\title{
Reduced miRNA-218 expression in pancreatic cancer patients as a predictor of poor prognosis
}

\author{
B.-S. Li, H. Liu and W.-L. Yang \\ Department of Gastrointestinal and Pancreatic Surgery, \\ The Third Xiangya Hospital of Central South University, Changsha, Hunan, China \\ Corresponding author: W.-L. Yang \\ E-mail: xy_yangwenlong@126.com \\ Genet. Mol. Res. 14 (4): 16372-16378 (2015) \\ Received August 28, 2015 \\ Accepted October 7, 2015 \\ Published December 9, 2015 \\ DOI http://dx.doi.org/10.4238/2015.December.9.5
}

ABSTRACT. microRNA-218 (miR-218) is a vertebrate-specific miRNA that plays a crucial role in tumorigenesis and tumor progression. This study analyzed the miR-218 expression level and clinical significance in pancreatic cancer. One hundred and seven pairs of pancreatic cancer and adjacent normal tissues were analyzed by quantitative reversetranscriptase polymerase chain reaction. The correlation between miR218 expression and clinicopathological characters was determined by the two-sample Student $t$-test. The survival correlations were analyzed by the Kaplan-Meier method and Cox proportional hazards model. The relative expression of miR-218 in pancreatic cancer tissues $(2.63 \pm 1.59)$ was significantly lower than that in matched noncancerous pancreatic tissues $(6.52 \pm 2.50, P<0.001)$. The low expression of miR-218 in the pancreatic cancer tissues were strongly correlated with the TNM classification $(P=$ $0.02)$, distant metastasis $(P=0.001)$, and tumor differentiation $(P=0.003)$. The low level of miR-218 expression was significantly correlated with the shorter overall survival time of pancreatic cancer patients (5-year overall survival rate: 7.5 vs $34.9 \%$; log-rank test: $P<0.001)$. Multivariate analyses confirmed that a low level of miR-218 expression was an independent 
predictor of poor prognosis in pancreatic cancer patients (Hazard ratio: 7.24; 95\% confidence interval: 2.01-18.28; $P=0.007$ ). Our findings suggested a significant downregulation in the expression of miR-218; this might have considerable potential value in the prognosis for pancreatic cancer.

Key words: MicroRNA-218; Prognosis; Pancreatic cancer

\section{INTRODUCTION}

Pancreatic cancer is a lethal malignancy (Siegel et al., 2013) that is responsible for an estimated 227,000 deaths per year worldwide; it is also the fourth leading cause of cancer-related deaths in the USA (Raimondi et al., 2009). Only $10-20 \%$ of the diagnosed pancreatic cancer is resectable, and its 5 -year survival rate is only $5 \%$; this can be attributed to its high recurrence rate, despite the multimodality treatment (Hidalgo, 2010; Vincent et al., 2011).

Mature micro-RNA (miRNA) are small (20 21 nucleotides in length) endogenous noncoding RNA that regulate the expression of target genes at the post-transcriptional level through the degradation of transcripts and inhibition of translation, by binding to the 3 '-untranslated region (UTR) of target messenger RNA (mRNA) (Bartel, 2004). miRNA have been shown to be involved in various critically important physiological processes, such as cell proliferation, division, differentiation, and apoptosis, tumorigenesis, hematopoiesis, and patterning of the nervous system (Ambros, 2004). miRNA signatures have been associated with the diagnosis, staging, progression, and prognosis of human cancer, and its response to treatment (Esquela-Kerscher and Slack, 2006).

microRNA-218 (miR-218) is a vertebrate-specific miRNA that has been predicted and experimentally confirmed to play a crucial role in tumorigenesis and tumor progression by regulating the expression of potential targets (Hassan et al., 2012). Recent studies have indicated that miR218 is down-regulated in various types of cancer, including cervical cancer, gastric cancer, lung cancer, colon cancer, prostate cancer, and bladder cancer (Davidson et al., 2010; Song et al., 2010; Tie et al., 2010; Uesugi et al., 2011; Leite et al., 2011; Li et al., 2012a,b; Chiyomaru et al., 2012; He et al., 2012). However, the level of expression of miR-218 and its clinical significance in pancreatic cancer has not been investigated. This study compares the level of expression of miR-218 between pancreatic cancer cells and normal adjacent pancreatic tissues, in addition to investigating its clinical significance and prognostic value.

\section{MATERIAL AND METHODS}

\section{Patients and tumor tissue samples}

This study was approved by the Ethics Committee on Human Research of the Third Xiangya Hospital of Central South University. Informed consent was obtained from all of the recruited patients. One hundred and seven human pancreatic cancer tissues and matched normal adjacent pancreatic tissues were surgically obtained from the recruited patients; the surgeries were performed at the Department of Gastrointestinal and Pancreatic Surgery of the Third Xiangya Hospital of Central South University between March 2008 and January 2013. The diagnosis was based on pathological evidence; the specimens were immediately snap-frozen and stored at $-80^{\circ} \mathrm{C}$ for future miR-218 extraction. None of the patients received chemotherapy or radiotherapy prior to the surgical excision. The clinicopathological characteristics of the pancreatic cancer patients are summarized in Table 1. 


\section{Quantitative real-time polymerase chain reaction (PCR)}

Total RNA was extracted from the pancreatic cancer tissues and matched normal adjacent pancreatic tissues, by homogenization of the tissues with TRIzol reagent (Invitrogen, Carlsbad, California, USA) according to the manufacturer protocols. Primers for miR-218 and the endogenous control U6 snRNA were obtained from Applied Biosystems (Foster City, CA, USA). The concentration and purity of RNA was determined spectrophotometrically using NanoDrop ND-1000 (NanoDrop Technologies, Wilmington, DE, USA). cDNA was generated using the PrimeScript RT reagent kit (TaKaRa Bio Inc., Dalian, China) in a $20 \mu \mathrm{L}$ reaction mixture (final volume), containing $0.5 \mu \mathrm{RNA}$, $0.5 \mu \mathrm{L}$ Prime-Script RT enzyme mix, $4 \mu \mathrm{L} 5 \mathrm{X}$ PrimeScript buffer, and $1 \mu \mathrm{L}$ RT primer; cDNA was generated by incubating this reaction mixture at $42^{\circ} \mathrm{C}$ for $60 \mathrm{~min}$ and $85^{\circ} \mathrm{C}$ for $5 \mathrm{~min}$. The $\mathrm{miR}-218$ expression was evaluated by quantitative real-time PCR using SYBR Premix Ex Taq (Takara Bio Inc), and measured in a LightCycler 480 system (Roche Diagnostics, Basel, Switzerland). The reaction conditions were set as follows: denaturation at $95^{\circ} \mathrm{C}$ for $10 \mathrm{~min}$, followed by 45 cycles of denaturation at $95^{\circ} \mathrm{C}$ for $15 \mathrm{~s}$, annealing at $60^{\circ} \mathrm{C}$ for $30 \mathrm{~s}$, and extension at $72^{\circ} \mathrm{C}$ for $1 \mathrm{~min}$. The miRNA expression was relatively quantified by $2^{-\triangle \Delta C T}$ method. All raw data was presented as the relative quantity of target miRNA, normalized against the U6 snRNA, and relative to a calibrator sample.

\section{Statistical analysis}

The expression level of miR-218 was compared between pancreatic cancer tissues and matched normal adjacent pancreatic tissues using the two-sample Student $t$-test. The relationship between miR-218 expression and the clinicopathological parameters was evaluated by the $x^{2}$ test. The survival rates for miR-218 expression were estimated using the Kaplan-Meier method, and the difference in survival curves were calculated using the log-rank test. The survival data was evaluated using a multivariate Cox regression analysis. All statistical analyses were performed on an SPSS v.17.0 software platform for Windows (SPSS Inc., Chicago, IL, USA). P values $<0.05$ were considered to indicate statistical significance.

\section{RESULTS}

\section{Comparison of miR-218 expression levels between pancreatic cancer tissues and matched normal adjacent pancreatic tissues}

miR-218 was detected in all 107 pairs of pancreatic cancer and matched non-cancerous pancreatic tissue samples by qRT-PCR. The relative expression of miR-218 in pancreatic cancer tissues $(2.63 \pm 1.59)$ was significantly lower than that in matched noncancerous pancreatic tissues $(6.52 \pm 2.50, \mathrm{P}<0.001$; Figure 1$)$. The 107 pancreatic cancer patients were divided into a low expression group $(\mathrm{N}=54)$ and a high expression group $(\mathrm{N}=53)$, based on the median expression level of miR-218.

\section{Correlation between the level of miR-218 expression and clinicopathological factors}

The association between miR-218 expression and the clinicopathological variables in pancreatic cancer patients are summarized in Table 1. The low expression of miR-218 in the 
pancreatic cancer tissues was strongly correlated with the TNM classification $(P=0.02)$, distant metastasis $(P=0.001)$, and tumor differentiation $(P=0.003)$. However, the level of miR-218 expression in pancreatic cancer tissues was not associated with the age, gender, location, tumor size, and lymphatic invasion ( $P>0.05)$.

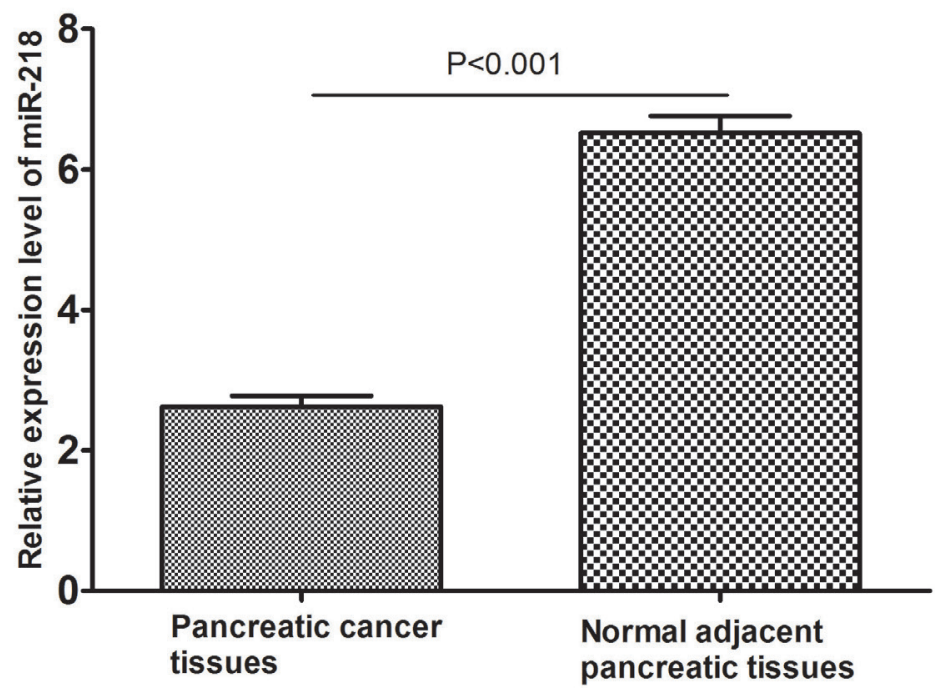

Figure 1. Expression level of the miR-218 in pancreatic cancer tissues and matched noncancerous pancreatic tissues.

\begin{tabular}{|c|c|c|c|c|}
\hline \multirow[t]{2}{*}{ Parameters } & \multirow[b]{2}{*}{ Number of cases } & \multicolumn{2}{|c|}{ miR-218 expression } & \multirow[b]{2}{*}{$P$ value } \\
\hline & & Low & High & \\
\hline \multicolumn{5}{|l|}{ Sex } \\
\hline Male & 68 & 35 & 33 & \multirow[t]{2}{*}{0.75} \\
\hline Female & 39 & 19 & 20 & \\
\hline \multicolumn{5}{|l|}{ Age (years) } \\
\hline$<65$ & 51 & 23 & 28 & \multirow[t]{2}{*}{0.27} \\
\hline$\geq 65$ & 56 & 31 & 25 & \\
\hline \multicolumn{5}{|l|}{ Location } \\
\hline Head, urinate & 79 & 39 & 40 & \multirow[t]{2}{*}{0.82} \\
\hline Body, tail & 28 & 15 & 13 & \\
\hline \multicolumn{5}{|l|}{ Tumor size (cm) } \\
\hline$<2$ & 36 & 16 & 20 & \multirow[t]{2}{*}{0.42} \\
\hline$\geq 2$ & 71 & 38 & 33 & \\
\hline \multicolumn{5}{|l|}{ Lymphatic invasion } \\
\hline Positive & 58 & 36 & 22 & \multirow[t]{2}{*}{0.06} \\
\hline Negative & 49 & 18 & 31 & \\
\hline \multicolumn{5}{|l|}{ Distant metastasis } \\
\hline Positive & 11 & 10 & 1 & \multirow[t]{2}{*}{0.001} \\
\hline Negative & 96 & 44 & 52 & \\
\hline \multicolumn{5}{|l|}{ TNM stage } \\
\hline $1 / I I$ & 83 & 48 & 35 & \multirow[t]{2}{*}{0.02} \\
\hline III/IV & 24 & 6 & 18 & \\
\hline \multicolumn{5}{|c|}{ Tumor differentiation } \\
\hline High/moderate & 39 & 4 & 35 & \multirow[t]{2}{*}{0.003} \\
\hline Poor & 68 & 50 & 18 & \\
\hline
\end{tabular}




\section{Association between miR-218 expression and survival in pancreatic cancer patients}

The overall survival curves of pancreatic cancer patients were charted using the KaplanMeier method. The results revealed a significant correlation between the shorter overall survival time of pancreatic cancer patients and the low expression level of miR-218 (5 year overall survival rate, 7.5 vs $34.9 \%$; log-rank test, $\mathrm{P}<0.001$; Figure 2). Multivariate analysis using the Cox proportional hazards model confirmed low miR-218 expression level to be an independent predictor of poor prognosis in pancreatic cancer patients (Hazard ratio, 7.24; 95\% confidence interval $(\mathrm{Cl}), 2.01$ 18.28; $\mathrm{P}=0.007$; Table 2 ).

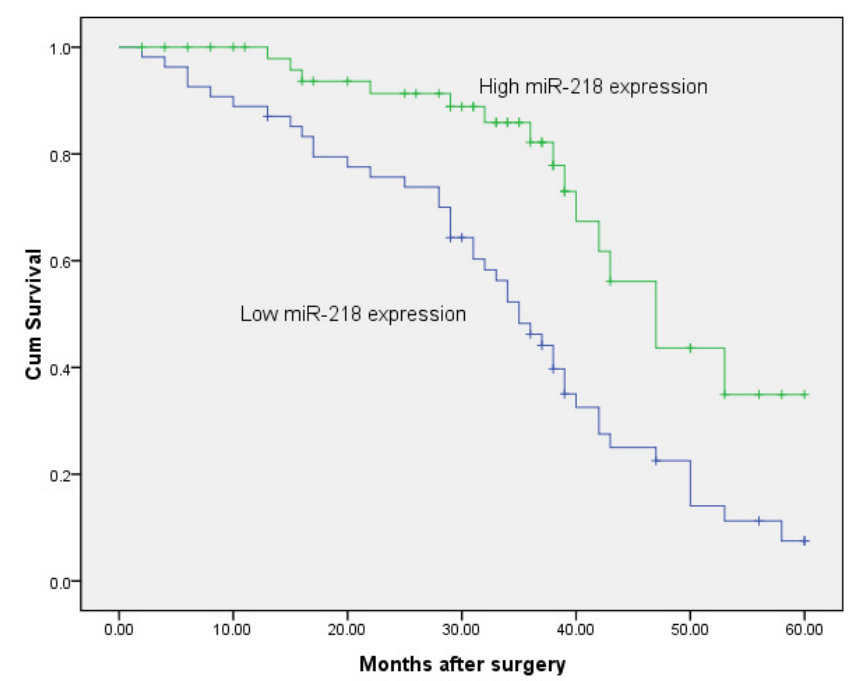

Figure 2. Kaplan-Meier survival analysis by miR-218 expression. Low expression level of miR-218 was significantly correlated with the shorter overall survival time of pancreatic cancer patients ( 5 year overall survival rate: 7.5 vs $34.9 \%$; log-rank test: $P<0.001)$.

Table 2. Multivariate Cox hazards model analysis for prognostic factors.

\begin{tabular}{llcc}
\hline Variable & Subset & Hazard ratio & $95 \% \mathrm{Cl}$ \\
\hline Sex & Male vs female & 1.49 & $0.82-1.14$ \\
Age (years) & $\geq 65$ vs $<65$ & 0.87 & $0.59-3.88$ \\
Location & Head, urinate vs body, tail & 1.52 & $0.61-1.91$ \\
Tumor Size (cm) & $\geq 2$ vs <2 & 1.87 & $0.78-6.46$ \\
Lymphatic invasion & Positive vs negative & 2.67 & 0.31 \\
Distant metastasis & Positive vs negative & 4.76 & $0.56-9.74$ \\
TNM stage & I/II vs III/IV & 2.99 & 0.11 \\
Tumor differentiation & Well-moderate vs poor & 2.97 & $1.49-16.56$ \\
miR-218 expression & High vs low & 7.24 & $0.97-7.45$ \\
\hline
\end{tabular}

\section{DISCUSSION}

Pancreatic carcinogenesis is a multi-step process involving multiple genetic and epigenetic alterations. Although many therapeutic strategies have been applied to treat this disease, the survival rate of advanced pancreatic cancer has remained unchanged over the past 
few decades. Accurate prediction of the prognosis of each individual pancreatic cancer patient is of great importance, and molecular biomarkers functioning as prognostic factors could be useful in determining an individualized treatment plan for each pancreatic cancer patient (Hurwitz et al., 1992). However, the biomarkers that are currently in use are not satisfactory; therefore, there is a need for the identification and development of additional markers to fine-tune this process (Winter et al., 2013). miRNA, which function as key post-transcription regulators, are involved in a wide variety of biological processes, including cell growth, development, proliferation, differentiation, and death (Esquela-Kerscher and Slack, 2006). The differential expression of miRNA among normal and cancerous tissues, and the significant correlation between specific expression and prognosis, indicate miRNA to be determinants of certain clinical outcomes.

Recent studies have demonstrated that miR-218 is downregulated in various types of cancer, including cervical cancer, gastric cancer, lung cancer, colon cancer, prostate cancer, and bladder cancer (Davidson et al., 2010; Song et al., 2010; Tie et al., 2010; Leite et al., 2011; Uesugi et al., 2011; Li et al., 2012a; Li et al., 2012b; Chiyomaru et al., 2012; He et al., 2012). However, the level of miR0218 expression, and its clinical significance in pancreatic cancer, remains to be investigated. In this study, we observed a significantly lower relative expression of miR-218 in pancreatic cancer tissues compared to that in matched noncancerous pancreatic tissues. The low expression of miR-218 in the pancreatic cancer tissues was strongly correlated with the TNM classification, distant metastasis, and tumor differentiation, as well as the shorter overall survival time $(\mathrm{P}<0.001)$ of pancreatic cancer patients. Multivariate analyses using the Cox proportional hazards model confirmed low miR-218 expression level to be an independent predictor of poor prognosis in pancreatic cancer patients (Hazard ratio, 7.24; 95\%Cl: 2.01-18.28; $\mathrm{P}=0.007$ ). Empirically, a HR > 1.5 is considered to be a strong prognostic factor (Hayes et al., 2001). Therefore, miR-218 might have considerable potential as a prognosis factor for pancreatic cancer.

miR-218 functions as a tumor suppressor gene in various types of cancer, targeting multiple genes and regulating biological processes (Song et al., 2010; Tie et al., 2010; Uesugi et al., 2011; He et al., 2012; Li et al., 2012b; Yamamoto et al., 2013). Overexpression of miR-218 is known to inhibit cancer cell proliferation, invasion, and metastasis, and promote cancer cell apoptosis. Li et al. (2012b) reported that the overexpression of miR-218 reduced human cervical cancer HeLa cell proliferation, and induced cell apoptosis through the AKT-mTOR signaling pathway. In addition, forced miR0218 overexpression was observed to suppress tumor growth in a HeLa cell orthotopic mouse model. In addition, miR-218 effected increased chemosensitivity to cisplatin (CDDP) in vitro (Li et al., 2012b). Wei et al have reported that miR-218 is downregulated in melanoma cells. miR-218 regulates the proliferation, migration, and invasion of melanoma cell lines A375 and SKMEL-2 by targeting CIP2A and BMI1, indicating the pivotal role played by miR-218 in melanoma development (Wei et al., 2014). A previous study conducted by Nishikawa et al. (2014) revealed a significant inhibition of cancer cell migration and invasion upon restoration of miR-218 in prostate cancer cell lines (PC3 and DU145). Gene expression data and in silico analyses identified LIM and SH3 protein 1 (LASP1) as a potential target for miR-218 regulation. LASP1 is a cytoskeletal scaffold protein that plays a critical role in cytoskeletal organization and cell migration. In addition, luciferase reporter assays revealed that LASP1 expression was directly regulated by miR-218. Moreover, down-regulation of the LASP1 gene significantly inhibited cell migration and invasion in cancer cells, and LASP1 expression was upregulated in cancer tissues. They concluded that the loss of tumor-suppressive miR-218 enhanced cancer cell migration and invasion in PC3, through direct regulation of LASP1 (Nishikawa et al., 2014). However, miR-218-regulated cancer pathways and target genes involved in pancreatic cancer development and metastasis have not been 
investigated so far. Therefore, further studies are required to clarify its role in pancreatic cancer.

In conclusion, lower expression levels of miR-218 were observed in pancreatic cancer tissues, compared to matched noncancerous pancreatic tissues; the low miR-218 expression level was significantly correlated with shorter overall survival time of pancreatic cancer patients. miR218 could, therefore, be a useful prognostic biomarker for pancreatic cancer.

\section{Conflicts of interest}

The authors declare no conflict of interest.

\section{REFERENCES}

Ambros V (2004). The functions of animal microRNAs. Nature 431: 350-355.

Bartel DP (2004). MicroRNAs: Genomics, Biogenesis, Mechanism, and Function. Cell 116: 281-297.

Chiyomaru T, Enokida H, Kawakami K, Tatarano S, et al. (2012). Functional role of LASP1 in cell viability and its regulation by microRNAs in bladder cancer. Urol. Oncol. 30: 434-443.

Davidson MR, Larsen JE, Yang IA, Hayward NK, et al. (2010). MicroRNA-218 is deleted and downregulated in lung squamous cell carcinoma. PloS One 5: e12560.

Esquela-Kerscher A and Slack FJ (2006). Oncomirs - microRNAs with a role in cancer. Nat. Rev. Cancer 6: 259-269.

Hassan MQ, Maeda $\mathrm{Y}$, Taipaleenmaki H, Zhang W, et al. (2012). miR-218 directs a Wnt signaling circuit to promote differentiation of osteoblasts and osteomimicry of metastatic cancer cells. J. Biol. Chem. 287: 42084-42092.

Hayes DF, Isaacs $C$ and Stearns $V(2001)$. Prognostic factors in breast cancer: current and new predictors of metastasis. $J$. Mammary Gland Biol. Neoplasia 6: 375-392.

He X, Dong Y, Wu CW, Zhao Z, et al. (2012). MicroRNA-218 inhibits cell cycle progression and promotes apoptosis in colon cancer by downregulating BMI1 polycomb ring finger oncogene. Mol. Med. 18: 1491-1498.

Hidalgo M (2010). Pancreatic cancer. N. Engl. J. Med. 362: 1605-1617.

Hurwitz M, Sawicki M, Samara G and Passaro E Jr. (1992). Diagnostic and prognostic molecular markers in cancer. Am. J. Surg. 164: 299-306.

Leite KR, Sousa-Canavez JM, Reis ST, Tomiyama AH, et al. (2011). Change in expression of miR-let7c, miR-100, and miR218 from high grade localized prostate cancer to metastasis. Urol. Oncol. 29: 265-269.

Li BS, Zhao YL, Guo G, Li W, et al. (2012a). Plasma microRNAs, miR-223, miR-21 and miR-218, as novel potential biomarkers for gastric cancer detection. PloS One 7: e41629.

Li J, Ping Z and Ning H (2012b). MiR-218 impairs tumor growth and increases chemo-sensitivity to cisplatin in cervical cancer. Int. J. Mol. Sci. 13: 16053-16064.

Nishikawa R, Goto Y, Sakamoto S, Chiyomaru T, et al. (2014). Tumor-suppressive microRNA-218 inhibits cancer cell migration and invasion via targeting of LASP1 in prostate cancer. Cancer Sci. 105: 802-811.

Raimondi S, Maisonneuve P and Lowenfels AB (2009). Epidemiology of pancreatic cancer: an overview. Nat. Rev. Gastroenterol Hepatol. 6: 699-708.

Siegel R, Naishadham D and Jemal A (2013). Cancer statistics, 2013. CA Cancer J. Clin. 63: 11-30.

Song L, Huang Q, Chen K, Liu L, et al. (2010). miR-218 inhibits the invasive ability of glioma cells by direct downregulation of IKK-beta. Biochem. Biophys. Res. Commun. 402: 135-140.

Tie J, Pan Y, Zhao L, Wu K, et al. (2010). MiR-218 inhibits invasion and metastasis of gastric cancer by targeting the Robo1 receptor. PLoS Genet. 6: e1000879.

Uesugi A, Kozaki K, Tsuruta T, Furuta M, et al. (2011). The tumor suppressive microRNA miR-218 targets the mTOR component Rictor and inhibits AKT phosphorylation in oral cancer. Cancer Res. 71: 5765-5778.

Vincent A, Herman J, Schulick R, Hruban RH, et al. (2011). Pancreatic cancer. Lancet 378: 607-620.

Wei Y, Du Y, Chen X, Li P, et al. (2014). Expression patterns of microRNA-218 and its potential functions by targeting CIP2A and BMI1 genes in melanoma. Tumour Biol. 35: 8007-15.

Winter JM, Yeo CJ and Brody JR (2013). Diagnostic, prognostic, and predictive biomarkers in pancreatic cancer. J. Surg. Oncol. 107: 15-22.

Yamamoto N, Kinoshita T, Nohata N, Itesako T, et al. (2013). Tumor suppressive microRNA-218 inhibits cancer cell migration and invasion by targeting focal adhesion pathways in cervical squamous cell carcinoma. Int. J. Oncol. 42: 1523-1532. 